Focus

\section{Hemotherapy with New Blood Products (Issue 2)}

Coordination: Korte, W. (St. Gallen); Zeerleder, S.S. (Amsterdam)

\section{Hemovigilance (Issue 3)}

Coordination: Mansouri Taleghani, B. (Bern); Heuft, H.-G. (Hanover)

\section{Optimized Antigen Matching - Chances and Challenges}

in Molecular Times (Issue 4)

Coordination: Gassner, A. (Schlieren); Wagner, F.F. (Springe)

Granulocytes - from Basic Research to Clinical Use (Issue 5)

Coordination: Bugert, P. (Mannheim); Schulze, T. (Springe)

\section{Editorials}

284 Hemotherapy with New Blood Products Korte, W. (St. Gallen); Zeerleder, S.S. (Amsterdam)

3148 Hemovigilance Heuft, H.-G. (Hanover), Mansouri Taleghani, B. (Bern)

4216 Optimized Antigen Matching - Chances and Challenges in Molecular Times Gassner, C. (Schlieren); Wagner, F.F. (Springe)

5288 Granulocytes: From Basic Research to Clinical Use Schulze, T. (Springe); Bugert, P. (Mannheim)

Research Articles

15 ABO Mistyping of cis-AB Blood Group by the Automated Microplate Technique

Chun, S.; Ryu, M.R.; Cha, S.-Y.; Seo, J.-Y., Cho, D. (Seoul)

113 Robust Production of Cytomegalovirus pp65-Specific T Cells Using a Fully Automated IFN- $\gamma$ Cytokine Capture System

Kim, N.; Nam, Y.-S.; Im, K.-I.; Lim, J.-Y.; Jeon, Y.-W.; Song, Y.; Lee, J.W.; Cho, S.-G. (Seoul)

124 Platelet Count before Peripheral Blood Stem Cell Mobilization Is Associated with the Need for Plerixafor But Not with the Collection Result Baertsch, M.-A.; Kriegsmann, K.; Pavel, P.; Bruckner, T.; Hundemer, M.; Kriegsmann, M.; Ho, A.D.; Goldschmidt H. (Heidelberg); Wuchter, P. (Mannheim)
133 Reactive Oxygen Species Generated by CD45- Cells Distinct from Leukocyte Population in Platelet Concentrates Is Correlated with the Expression and Release of Platelet Activation Markers during Storage Ghasemzadeh, M.; Hosseini, E.; Shahbaz Ghasabeh A.; Mousavi Hosseini K. (Tehran)

142 Post-Operative Iron Carboxymaltose May Have an Effect on Haemoglobin Levels in Cardiothoracic Surgical Patients on the ICU - an Observational Pilot Study about Anaemia Treatment with Intravenous Iron Peters, F.; Eveslage, M.; Gallitz I.; Wempe, C. (Münster), Meybohm, P. (Frankfurt/M.), Van Aken, H.; Steinbicker A. (Münster)

148 Association of $\boldsymbol{I L - 6}$ C-572G Gene Polymorphism with Anti-E Production Lin, J.-S.; Lee, L.-H.; Liu, H.-M.; Chen, Y.-J.; Chiou, T.-J. (Taipei)

155 Interaction of Platelet-Derived Microparticles with a Human B-Lymphoblast Cell Line: A Clue for the Immunologic Function of the Microparticles Yari, F.; Motefaker, M.; Nikougoftar, M.; Khayati, Z. (Tehran)

$162 \mathrm{ABO}$, Rhesus, and Kell Antigens, Alleles, and Haplotypes in West Bengal, India

Basu, D. (Kolkata); Datta, S.S. (Durgapur); Montemayor, C. (Bethesda, MD); Bhattacharya, P.; Mukherjee, K. (Kolkata); Flegel, W.A. (Bethesda, MD)

2108 Which Infectious Blood Donors Could Be Identified by the Donor History Questionnaire? - Comparison of Blood Donors Infected with HIV or HCV with Notified Cases from General Population in Germany Preußel, K.; Offergeld, R. (Berlin)

2116 Incentives for Blood Donation: A Discrete Choice Experiment to Analyze Extrinsic Motivation Sadler, A. (Neubrandenburg); Shi, L. (Boston, MA), Bethge, S.; Mühlbacher, A. (Neubrandenburg)

2127 Peripartum Haemorrhage: Haemostatic Aspects of the New German PPH Guideline

Lier, H. (Cologne); von Heymann, C. (Berlin), Korte, W. (St. Gallen); Schlembach; D. (Berlin)

3151 Nationwide Implementation of Pathogen Inactivation for All Platelet Concentrates in Switzerland Jutzi, M.; Mansouri Taleghani, B.; Rueesch, M.; Amsler, L. (Bern); Buser, A. (Basel)

3158 Unmatched Type O RhD+ Red Blood Cells in Multiple Injured Patients Flommersfeld, S.; Mand, C. (Marburg); Kühne, C.A. (Hamburg); Bein, G. (Gießen); Ruchholtz, S. (Marburg); Sachs, U.J. (Marburg/Gießen)

\section{KARGER}

Fax +4976145207 14

Information@Karger.com

www.karger.com
(๑) 2018 S. Karger GmbH, Freiburg 
3162 Anti-D Alloimmunization after RhD-Positive Platelet Transfusion in RhD-Negative Women under 55 Years Diagnosed with Acute Leukemia: Results of a Retrospective Study

Villalba, A.; Santiago, M.; Freiria, C.; Montesinos, P.; Gomez, I.; Fuentes, C.; Rodriguez-Veiga, R.; Fernandez, J.M.; Sanz, G.; Sanz, M.A.; Carpio, N. (Valencia); Solves, P. (Madrid)

3167 Platelet Transfusion Induces Alloimmunization to D and Non-D Rhesus Antigens

Reckhaus, J.; Jutzi, M.; Fontana, S.; Bacher, V.U.; Vogt, M.; Daslakis, M.; Mansouri Taleghani, B. (Bern)

3173 RHD-Positive Alleles among D- C/E+ Individuals from India

Kulkarni, S.S.; Gogri, H.; Parchure, D.; Mishra, G.; Ghosh, K.; Rajadhyaksha, S.; Madkaikar, M. (Mumbai); Férec, C.; Fichou, Y. (Brest)

3179 Red Cell Alloantibody Screening: Comparative Analysis of Three Different Technologies

Orlando, N.; Bianchi, M.; Valentini, C.G.; Maresca, M.; Massini, G.; Putzulu, R.; Zini, G.; Teofili, L. (Rome)

3185 Characteristics of Extracellular Vesicles in Red Blood Concentrates Change with Storage Time and Blood Manufacturing Method Almizraq, R.J.; Holovati, J.L.; Acker, J.P. (Edmonton, AB)

4218 The Impact of Using Genotyped Reagent Red Blood Cells in Antibody Identification

Scharberg, E. (Baden-Baden); Rink, G.; Portegys, J. (Mannheim); Rothenberger, S.; Gillhuber, N.; Richter, E. (Baden-Baden); Bugert, P. (Mannheim)

4225 Extended Donor Typing by Pooled Capillary Electrophoresis: Impact in a Routine Setting Wagner F.F. (Springe); Doescher, A. (Oldenburg); Bittner, R.; Müller, T.H. (Springe)

4239 Low-Frequency Blood Group Antigens in Switzerland Gassner, C. (Schlieren); Degenhardt, F. (Kiel); Meyer, S. (Schlieren); Vollmert, C. (Hamburg); Trost, N.; Neuenschwander, K.; Merki, Y.; Portmann, C.; Sigurdardottir, S.; Zorbas, A.; Engström, C.; Gottschalk, J. (Schlieren); Amar el Dusouqui, S.; Waldvogel-Abramovski, S.; Rigal, E. (Geneva); Tissot, J.-D. (Lausanne); Tinguely, C. (Bern); Mauvais, S.M.; Sarraj, A. (Neuchatel); Bessero, D.; Stalder, M. (Sion); Infanti, L.; Buser, A. (Basel); Sigle, J. (Aarau); Weingand, T. (Luzern); Castelli, D. (Lugano); Braisch M.C.; Thierbach, J. (Sankt Gallen); Heer, S.; Schulzki, T. (Chur); Krawczak, M.; Franke, A. (Kiel); Frey, B.M. (Schlieren)

4252 Rh-Matched Transfusion through Molecular Typing for $\beta$-Thalassemia Patients Is Required and Feasible in Chinese

Shao, C.-P.; Zhao, C.-J.; Wu, C.-L- (Shenzhen); Xu, H. (Xi'an); Wang, X.-D. (Wuxi); Wu, X.-Y.; Yi, P.; Dang, X.-T. (Shenzhen)

5323 Low-Volume Leukapheresis in Non-Cytokine-Stimulated Donors for the Collection of Mononuclear Cells Anyanwu, A.; Sitzmann, N.; Hetjens, S.; Klüter, H.; Wuchter, P. (Mannheim)
5331 Towards a Regional Registry of Extended Typed Blood Donors: Molecular Typing for Blood Group, Platelet and Granulocyte Antigens

Portegys, J.; Rink, G.; Bloos, P. (Mannheim); Scharberg E.A. (Baden-Baden); Klüter, H.; Bugert, P. (Mannheim)

5341 Evaluation of the New Lateral Flow Card MDmulticard ${ }^{\circledR}$ Basic Extended Phenotype in Routine Clinical Practice Mayer, B. (Berlin); Müller, J. (Ulm); Candela-García M.-J. (Murcia); Manteau A.-C. (Valenciennes); Weinstock, C. (Ulm); Pruß A (Berlin)

5347 Vitamin E Analogue Protects Red Blood Cells against Storage-Induced Oxidative Damage Antosik, A.; Czubak, K.; Cichon, N.; Nowak, P.; Zbikowska, H. (Lodz)

6378 Complement Deposition and IgG Binding on Stored Red Blood Cells Are Independent of Storage Time Thielen, A.J.F.; Meulenbroek, E.M.; Baas, I.; Bruggen, R.; Zeerleder, S.S.; Wouters, D. (Amsterdam)

6385 Rotational Thromboelastometry Helps to Reduce Blood Product Consumption in Critically Ill Patients during Small Surgical Procedures at the Intensive Care Unit - a Retrospective Clinical Analysis and Literature Search Vymazal, T.; Astraverkhava, M.; Durila, M. (Prague)

6388 Human Platelet Antigens in Brazilian Multiethnic Populations: Occurrence of Regional Variation and Frequency in a Large Urban Center (Belo Horizonte)

Silva-Malta, M.C.F.; de Oliveira, L.G.T; Barreiros, L.F.; do Amaral, D.R.; Martins, M.L. (Belo Horizonte)

6397 MicroRNA Dysregulation Associated with Red Blood Cell Storage

Chen, X.; Xie, X.; Xing, Y.; Yang, X.; Yuan, Z.; Wei, Y. (Guangzhou)

6404 Perioperative Thromboelastometry for Adult Living Donor Liver Transplant Recipients with a Tendency to Hypercoagulability: A Prospective Observational Cohort Study

Kamel, Y.; Hassanin, A.; Ahmed, A.R.; Gad, E.; Afifi, M.; Khalil, M. (Sheeben El Kom); Görlinger, K. (Essen); Yassen, K. (Shebeen El Kom)

6413 The Blood Bag Plasticizer Di-2-Ethylhexylphthalate Causes Red Blood Cells to Form Stomatocytes, Possibly by Inducing Lipid Flip-Flop Melzak, K.A. (Eggenstein-Leopoldshafen); Uhlig, S. (Mannheim); Kirschhöfer, F.; Brenner-Weiss, G. (Eggenstein-Leopoldshafen); Bieback, K. (Mannheim)

6423 Usefulness of Non-Invasive Fetal RHD Genotyping towards Immunoprophylaxis Optimization Blanco, S.; Giacomi, V.S.; Slobodianiuk, L.G.; Frutos, M.C.; Carrizo, L.H.; Fanin, G.E.; Culasso, J.M.; Gallego S.V. (Córdoba)

6429 Efficacy of Antenatal Intravenous Immunoglobulin Treatment in Pregnancies at High Risk due to Alloimmunization to Red Blood Cells Mayer, B.; Hinkson, L.; Hillebrand, W.; Henrich, W.; Salama, A. (Berlin) 
Review Articles

286 Extended Half-Life Factor VIII and Factor IX

Preparations

Graf, L. (St. Gallen)

292 The Potential Close Future of Hemophilia Treatment Gene Therapy, TFPI Inhibition, Antithrombin Silencing, and Mimicking Factor VIII with an Engineered Antibody Korte, W.; Graf L. (St. Gallen)

298 Platelet Additive Solutions: A Review of the Latest Developments and Their Clinical Implications van der Meer, P.F.; de Korte, D. (Amsterdam)

4258 Optimized Antigen-Matched in Sickle Cell Disease Patients: Chances and Challenges in Molecular Times the Brazilian Way

Castilho, L. (Campinas); Dinardo, C.L. (Sao Paulo)

4264 Genotyping in Sickle Cell Disease Patients: The French Strategy

Floch, A.; Tournamille, C.; Chami, B.; Pirenne, F. (Créteil )

4271 Transfusion Support of Minority Patients: Extended Antigen Donor Typing and Recruitment of Minority Blood Donors

Khan, J. (Seattle, WA); Delaney, M. (Washington, DC)

5290 The Pathogenic Involvement of Neutrophils in Acute Respiratory Distress Syndrome and Transfusion-Related Acute Lung Injury

Rebetz, J.; Semple J.W.; Kapur, R. (Lund)

5300 Molecular Genetics of the Human Neutrophil Antigens Flesch B.K.; Reil, A. (Bad Kreuznach)

5311 Neonatal Alloimmune Neutropenia Porcelijn, L.; de Haas, M. (Amsterdam)

5318 An International Registry of Granulocyte Transfusions Pagano M.B. (Seattle, WA); Morton, S. (Birmingham); Cohn C.S (Minneapolis, MN); Gross, S. (La Plaine-Saint Denis); Kutner, J. (Sao Paulo); Lewin, A. (Montreal, QC); McCullough, J. (Minneapolis, MN); Schweitzer, I.; Tinmouth A.T. (Ottawa, ON); West, K. (Besthesda, MD); Stanworth S.J. (Oxford)

Systematic Review

3195 The Role of Autologous Platelet Concentrates in Alveolar Socket Preservation: A Systematic Review Annunziata, M.; Guida, L.; Nastri, L.; Piccirillo, A.; Sommese, L.; Napoli, C. (Naples)

5355 Mortality, Morbidity and Related Outcomes Following Perioperative Blood Transfusion in Patients with Major Orthopaedic Surgery: A Systematic Review Müller, S.; Oberle, D.; Drechsel-Bäuerle, U.; Pavel, J.; Keller-Stanislawski, B.; Funk M.B. (Langen)
Case Reports

2104 Recurrent Bleedings in Newborn: A Factor VII Deficiency Case Report Cattivelli, K.; Distefano, C. (Brescia), Bonetti, L.; Testa, S. (Cremona); Siboni, S.M. (Milan); Plebani, A. (Brescia); Poggiani, C. (Cremona)

6438 Fatal Acute Hemolytic Transfusion Reaction due to Anti-Wr Bahri, T.; de Bruyn, K.; Leys, R.; Weerkamp, F. (Rotterdam)

Commentary

167 Young Blood Rejuvenates Old Bodies: A Call for Reflection when Moving from Mice to Men Hofmann, B. (Oslo)

Forum

172,3205

News / Ticker

1 75, 2 137, 3 207, 4 277, 5 368, 6445

Meetings and Conferences

1 79, 2 141, $3211,4283,5371,6451$

Erratum

4224

Acknowledgement to Reviewers

6442

Contents of Vol. 45, 2018

6376

Supplement 1

51. Jahrestagung der Deutschen Gesellschaft für Transfusionsmedizin und Immunhämatologie (DGTI) 19.-21. September 2018, Lübeck ABSTRACTS

Gast-Herausgeber:

Prof. Dr. Siegfried Görg, Prof. Dr. Holger Hennig, Lübeck ISBN 978-3-318-06429-2 